\title{
REDES DE FORMACIÓN PROFESIONAL: GESTIÓN, ADMINISTRACIÓN Y EMPRENDIMIENTO DEL CONOCIMIENTO
}

\author{
VOCATIONAL TRAINING NETWORKS: \\ KNOWLEDGE MANAGEMENT, ADMINISTRATION \\ AND ENTREPRENEURSHIP
}

Recibido: 14 de julio de 2020

Evaluado: 23 de agosto de 2020

Aprobado: 12 de septiembre de 2020

\author{
Celia Yaneth Quiroz Campas* \\ Nova Southeastern University \\ Orcid: https://orcid.org/0000-0002-6068-1552
}

Cruz García Lirios

Universidad Autónoma del Estado de México

Orcid: https://orcid.org/0000-0002-9364-6796

Cómo citar este artículo: Quiroz Campas, C.Y., y Gracía Lirios, C. (2021). REDES DE FORMACIÓN PROFESIONAL: GESTIÓN, ADMINISTRACIÓN Y EMPRENDIMIENTO DEL CONOCIMIENTO. Revista Estrategia Organizacional, 10(1), https://doi.org/10.22490/25392786.4561

\footnotetext{
* Doctora en educación. Actualmente vinculada a la Red Académica Internacional e Interinstitucional de Investigación Multidisciplinar. Correo electrónico: Celia.quiroz@itson.edu.mx
} 


\title{
RESUMEN
}

Introducción: A partir de este modelo de red, el objetivo del presente estudio es establecer mediante análisis de discursos las relaciones entre periferias de entrada, nodos trans-periféricos entrantes, centrales periféricas, nodo central, nodo trans-periférico saliente y periferias de salida. Metodología: Se llevó a cabo un estudio exploratorio de corte cualitativo con entrevistas a profundidad y análisis del discurso de tres participantes en una red formativa profesional. Resultados: Los resultados muestran que la innovación en la red es incipiente y ello supone la emergencia de agentes que por su gestión, innovación y emprendimiento resultan ser los únicos productores de conocimiento que incentivan a estudiantes, practicantes o administrativos, pero no reciben influencia alguna de ellos durante la capacitación y supervisión de prácticas profesionales en las empresas que establecieron convenios de colaboración con la universidad pública. En referencia al estado del conocimiento los resultados de la presente investigación fueron discutidos.

Palabras clave: Red, nodo, clima, grupo, emprendimiento, administración.

\begin{abstract}
Introduction: Based on this network model, the objective of this study is to establish, through discourse analysis, the relationships between input peripheries, incoming trans-peripheral nodes, peripheral centrals, central node, outgoing trans-peripheral node and output peripheries. Methodology: A qualitative exploratory study was carried out with in-depth interviews and discourse analysis of three participants in a professional training network. Results: The results show that innovation in the network is incipient and this implies the emergence of agents that, due to their management, innovation and entrepreneurship, turn out to be the only producers of knowledge that encourage students, practitioners or administrators, but do not receive any influence from them during the training and supervision of professional practices in companies that established collaboration agreements with the public university. Regarding the state of knowledge, the results of the present investigation were discussed.
\end{abstract}

Keywords: Network, node, climate, group, entrepreneurship, administration. 


\section{INTRODUCCIÓN}

En el marco del desarrollismo organizacional y educativo, las redes de formación profesional son aquellas que transfieren la producción de conocimiento del centro a la periferia. En este modelo de gestión, innovación y emprendimiento, existen nodos trans-periféricos que diseminan habilidades, valores y saberes en función de los flujos de información que se pueden estructurar en entrada y salidas. El balance general de las diferencias entre las entradas y salidas de información determina el desarrollo de la red (véase figura 1).

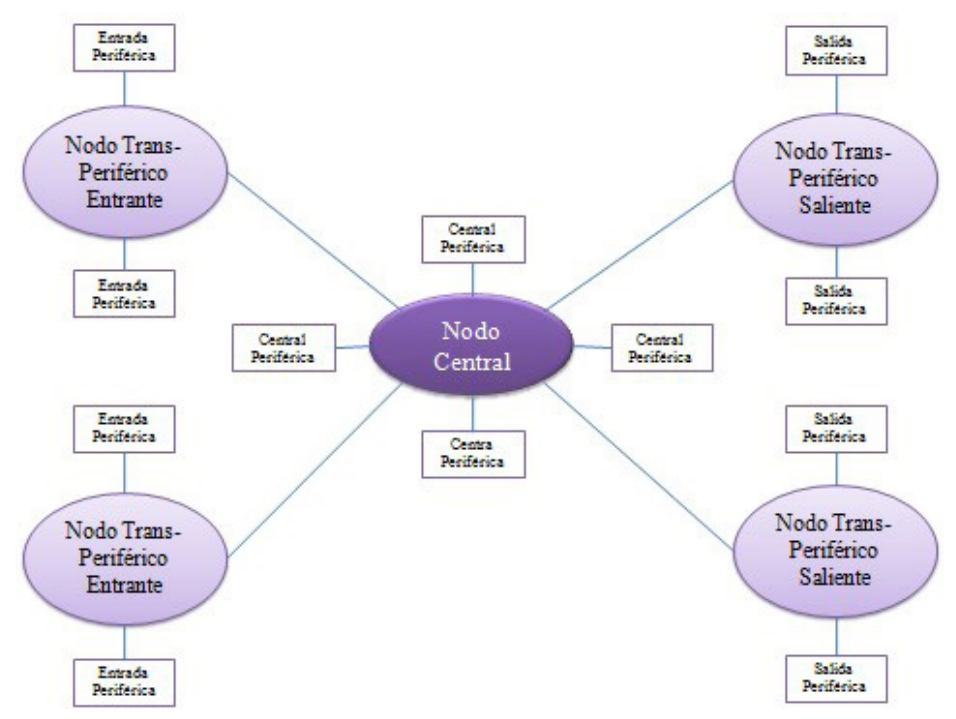

Figura 1. Modelo de red Fuente: Elaboración propia

A partir de este modelo de red, el objetivo del presente estudio es establecer mediante análisis de discursos las relaciones entre periferias de entrada, nodos trans-periféricos entrantes, centrales periféricas, nodo central, nodo trans-periférico saliente y periferias de salida (Arnau y Montané, 2010).

Para tal propósito, se especificaron agentes tales como; profesores, administrativos, estudiantes, practicantes, UAEM y NISSAN siguiendo el modelo de red y estructurando su participación en torno a gestión, innovación y emprendimiento derivados de convenios de prácticas profesionales y capacitación establecidos a partir del tercer semestre del plan de estudios vigente y con una duración de tres semestres que pueden ser vinculantes con el servicio social y prácticas orientadas a la elaboración de tesis o proyectos terminales (véase figura 2). 
El modelo especificado de red formativa cobra su relevancia considerando una red formativa desarrollada en la que los agentes interactúan unos con otros enalteciendo el nodo central de innovación que supone una formación profesional gestora a emprendedora del conocimiento (véase figura 3).

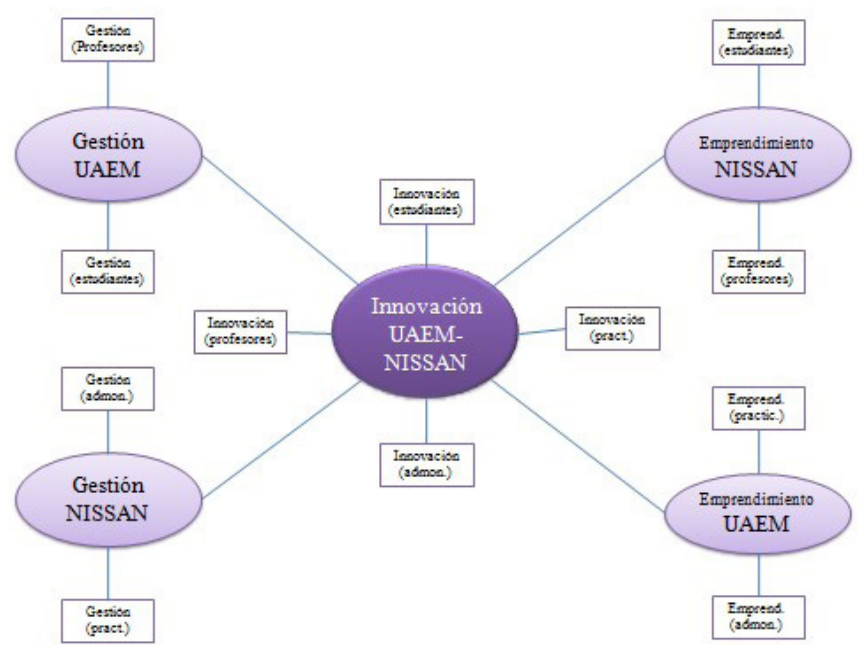

Figura 2. Red de formación profesional UAEM-NISSAN

Fuente: Elaboración propia

En este modelo de red formativa desarrollada las oportunidades, capacidades y responsabilidades son indicativas de satisfacciones de vida porque derivan de climas organizacionales de confianza, compromiso y cooperación, aunque sólo en un sentido que va de la periferia entrante a la periferia saliente inhibiendo la emergencia de otro nodo central, o bien, se trata de un modelo de red formativa orientada a la demanda del mercado local, ya que las políticas de fomento empresarial son quienes financian la sinergia entre instituciones y empresas (Castel y Freundlich, 2010).

De este modo, el desarrollo local puede ser explicado desde los discursos que los agentes gestores, innovadores y emprendedores de conocimiento generan considerando alianzas estratégicas entre empresas trasnacionales y universidades (Castro y Martins, 2010).

En referencia a otros modelos tales como las franquicias en las que el clima laboral está determinado por el ajuste de la sucursal, en el modelo de red formativa del conocimiento la gestión antecede a la innovación porque se trata de nuevas formas de organización, discursos, productos o beneficios (Chinchilla y Cruz, 2010). 
La planificación de la red formativa no garantiza su desarrollo ya que la innovación más bien es una consecuencia de un clima laboral flexible en el que la creatividad de los agentes constituye una gestión paralela a la establecida (Fuentes y Sánchez, 2010).

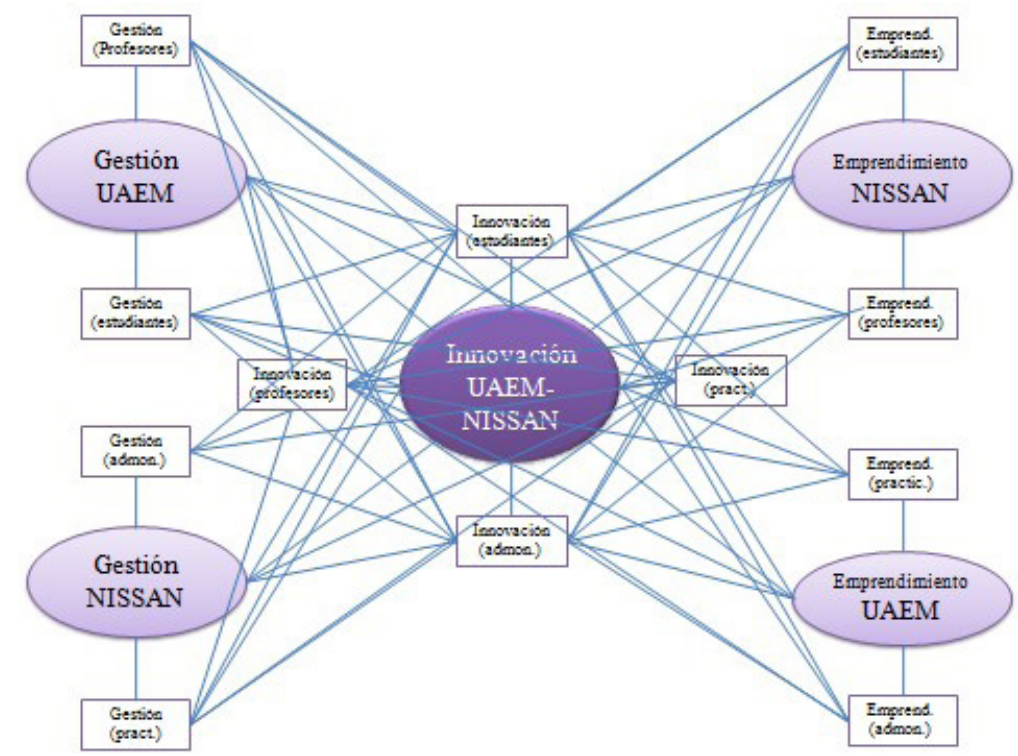

Figura 3. Red de formación profesional desarrollada

Fuente: Elaboración propia

Es el caso de quienes reformulan los procesos de gestión y diseminan las innovaciones de un modo tal que fomentan el emprendimiento de nuevos agentes como los son estudiantes de primer o segundo semestre, profesores de nuevo ingreso, administrativos ascendidos o practicantes que han tenido una continuidad en sus funciones mientras desarrollan sus proyectos de titulación.

Ahora bien, el estado del conocimiento del modelo de red formativa ha establecido como nodos trans-periféricos a la gestión del conocimiento y al clima organizacional. En el primer caso, el liderazgo es indicativo de la gestión, pero en referencia a la cultura organizacional (Omar, 2010). La gestión del conocimiento parte del supuesto acerca de que la cultura laboral refleja la gestión del conocimiento. Es decir, la noción de cultura es indicativo de una gestión, ya que una cultura de resistencia o apertura a la tecnología deriva en una gestión del control o la innovación (Galindo y Echavarría, 2011). 
Por su parte el clima organizacional, antecedente de la innovación, fundamenta sus relaciones de tarea en la confianza y el compromiso. El clima laboral que refleja confianza y disemina compromiso está enlazado con la innovación en tanto que las relaciones son asimétricas o simétricas. Un incremento en los conflictos genera cambios y un aumento en la cooperación produce competencias en un esquema de demandas y recursos (Carreón, 2014).

El compromiso sustenta el clima laboral necesario para la innovación estribe en nuevas formas de cooperación, nuevas competencias, nuevos modos de producción, o bien, nuevas estrategias de mediación de conflictos (Tayo y Adeyemi, 2012).

Ambos nodos trans-periféricos, gestión y clima al estar vinculados al nodo central de la innovación anticipan la satisfacción laboral y el emprendimiento (Orantes, 2011), pero no siempre los elementos periféricos salientes están relacionados con la confianza (Carreón, 2013).

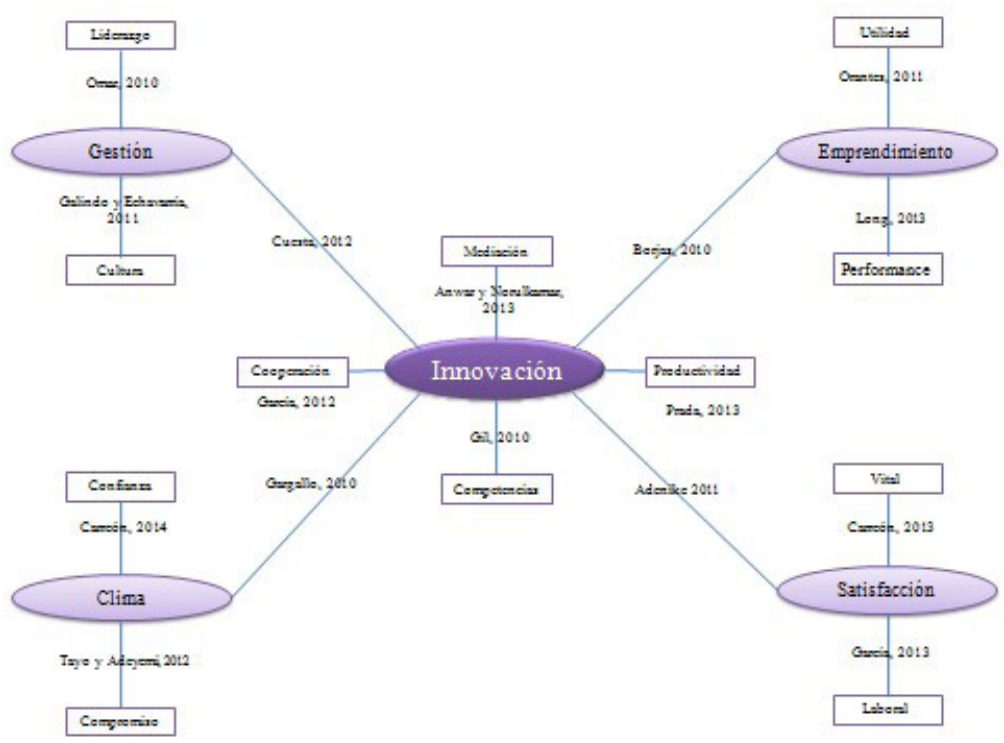

Figura 4. Estado del conocimiento de la red formativa

Fuente: Elaboración propia

Una red formativa profesional puede tener climas laborales de confianza y compromiso, pero la entrada de estos factores no garantiza la ejecución del emprendimiento, sólo su utilidad (Long, 2013). Del mismo modo, la mediación de conflictos podría coadyuvar a la satisfacción laboral o 
vital, empero ambos indican más bien nuevas formas de relaciones (Adenike, 2011). El desarrollo de un modelo formativo; gestor, innovador y emprendedor más bien dependerá de las competencias; valores, habilidades y conocimientos (Gil, 2010).

En síntesis, el modelo de red formativa: gestora, innovadora y emprendedora puede ser utilizado con la finalidad de potenciar los convenios entre UAEM y NISSAN considerando que el intercambio de información es permanente y ininterrumpido. El balance entre la entrada de información para su transformación en nuevos modos y formas de emprendimiento es observable en los discursos al momento en que quienes han participado en una red formativa profesional manejan conceptos relativos a la gestión, innovación y emprendimiento al momento de recordar la capacitación y el seguimiento de las prácticas profesionales y servicios sociales (Galindo y Echavarría, 2011).

¿Cómo pueden registrarse estos conceptos de manera tal que permitan establecer un balance entre las entradas de información y salidas de conocimientos aplicados a un proyecto académico que las universidades esperan dirigir hacia empresas con la finalidad de reducir las brechas de conocimiento?

\section{METODOLOGÍA}

Diseño. Se llevó a cabo un estudio exploratorio de corte cualitativo.

Muestra. Se realizó una selección no probabilística con la técnica "efecto bola de nieve" para entrevistar a tres docentes de la Universidad Autónoma del Estado de Morelos que han formado a estudiantes mediante prácticas profesionales en Nissan SA de CV. El criterio de inclusión y exclusión fue la experiencia en el sistema de prácticas.

Instrumento. Se realizaron entrevistas a profundidad, las categorías de entrevista y análisis fueron gestión, innovación y emprendimiento considerando una revisión de conceptos y hallazgos (véase tabla 1). 
Tabla 1. Instrumento

\begin{tabular}{|c|c|c|c|c|c|}
\hline 寒 & 高 & Definición & 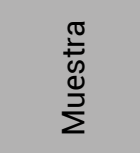 & Instrumento & Resultados \\
\hline $\begin{array}{l}\circ \\
\stackrel{\sim}{\sim}\end{array}$ & 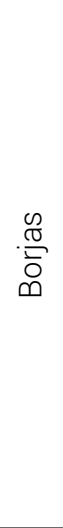 & $\begin{array}{l}\text { Emprendimiento. "(...) idea } \\
\text { innovadora, a través de la } \\
\text { creación de una organización } \\
\text { para explotar una oportunidad } \\
\text { detectada en el ambiente. } \\
\text { (...) una ventaja competitiva } \\
\text { que incluye una serie de } \\
\text { actividades tales como; } \\
\text { innovación y el desarrollo } \\
\text { de corporativas internas } \\
\text { y externas en productos y } \\
\text { procesos." (p. 151). }\end{array}$ & 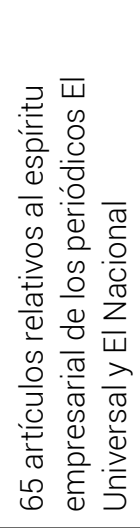 & $\begin{array}{l}\text { Matrices de } \\
\text { Análisis de } \\
\text { Contenido }\end{array}$ & $\begin{array}{l}\text { El espíritu empresarial fue una temática de } \\
\text { opinión pública social más que empresarial } \\
\text { u organizativa y estuvo relacionada con la } \\
\text { situación económica nacional así como } \\
\text { con la creatividad individual. Respecto } \\
\text { a los símbolos de representación social } \\
\text { el apoyo a microproyectos tuvo como } \\
\text { rasgos positivos la independencia del } \\
\text { Estado, empero los rasgos negativos } \\
\text { fueron considerados como iniciativas } \\
\text { intermitentes y efímeras. }\end{array}$ \\
\hline$\stackrel{\circ}{\circ}$ & 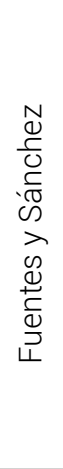 & $\begin{array}{l}\text { Emprendimiento. "(...) presenta } \\
\text { atributos como: originalidad } \\
\text { e innovación, moderada } \\
\text { aversión al riesgo; aceptación } \\
\text { de sus responsabilidades; } \\
\text { conocimiento de los } \\
\text { resultados de sus actos; } \\
\text { planificación en base al largo } \\
\text { plazo (...) necesidad de logro, } \\
\text { autoconfianza, optimismo, } \\
\text { creatividad y autonomía." (p. 3) }\end{array}$ & 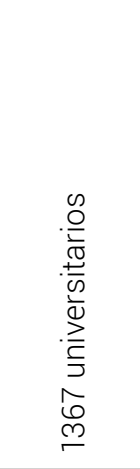 & $\begin{array}{l}\text { Cuestionario } \\
\text { sobre Perfil } \\
\text { Emprendedor } \\
\text { y escala } \\
\text { tipo Likert } \\
\text { de Atributos } \\
\text { Emprendedores }\end{array}$ & $\begin{array}{l}\text { No se encontraron diferencias } \\
\text { significativas entre hombres y mujeres } \\
\text { respecto a la expectativa e iniciativa de } \\
\text { emprender un proyecto, pero en cuanto } \\
\text { a los perfiles correspondientes al espíritu } \\
\text { emprendedor las mujeres consideran } \\
\text { que se requiere iniciativa, creatividad e } \\
\text { inventiva mientras que los hombres se } \\
\text { inclinan por los retos y las oportunidades } \\
\text { de las cuales se deriva su entusiasmo a los } \\
\text { desconocido. }\end{array}$ \\
\hline$\overline{\mathrm{N}}$ & 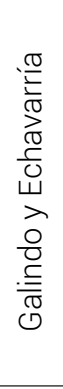 & $\begin{array}{l}\text { Emprendimiento. "(...) } \\
\text { capacidad para pensar } \\
\text { razonar y actuar centrada en } \\
\text { las oportunidades, planteada } \\
\text { con visión global y llevada a } \\
\text { cabo mediante un liderazgo } \\
\text { equilibrado y la gestión de un } \\
\text { riesgo calculado." (p. 88) }\end{array}$ & 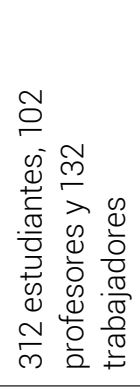 & $\begin{array}{l}\text { Escalas } \\
\text { de Rasgos } \\
\text { de Cultura } \\
\text { Empresarial }\end{array}$ & $\begin{array}{l}\text { Creatividad, innovación y valor de } \\
\text { conocimiento fueron similares en las } \\
\text { tres muestras, aunque los estudiantes } \\
\text { tienen una mayor propensión al riesgo } \\
\text { en comparación a los profesores, la } \\
\text { proactividad es el elemento menos } \\
\text { importante mientras que la búsqueda } \\
\text { de información se manifiesta como la } \\
\text { habilidad más importante. }\end{array}$ \\
\hline $\bar{\curvearrowright}$ & 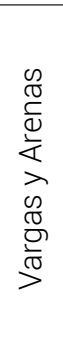 & $\begin{array}{l}\text { Emprendimiento. "(...) } \\
\text { habilidades necesarias para } \\
\text { que los jóvenes puedan crear, } \\
\text { liderar y sostener unidades de } \\
\text { negocio por cuenta propia." } \\
\text { (p. 26) }\end{array}$ & 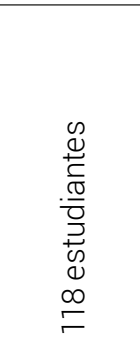 & $\begin{array}{l}\text { Cuestionario } \\
\text { de Habilidades } \\
\text { Emprendedoras } \\
\text { y Capacidades } \\
\text { de Gestión }\end{array}$ & $\begin{array}{l}86 \% \text { considera que la calidad educativa } \\
\text { es fundamental para las habilidades } \\
\text { empresariales, } 77 \% \text { están dispuestas a } \\
\text { reorientar sus proyectos ante un inminente } \\
\text { fracaso, } 90 \% \text { tiene una idea clara para su } \\
\text { futuro personal, } 72 \% \text { tiene un interés por lo } \\
\text { nuevo, } 90 \% \text { se consideran asertivos y } 60 \% \\
\text { están dispuestos a trabajar en equipo. }\end{array}$ \\
\hline
\end{tabular}




\begin{tabular}{|c|c|c|c|c|c|}
\hline 产 & $\begin{array}{l}\overline{0} \\
\frac{1}{2}\end{array}$ & Definición & 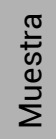 & Instrumento & Resultados \\
\hline$\stackrel{m}{\circ}$ & 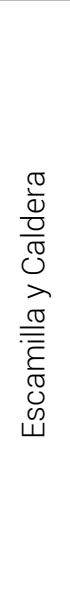 & $\begin{array}{l}\text { Percepción de oportunidad. } \\
\text { "La percepción que el individuo } \\
\text { tenga respecto al entorno } \\
\text { donde se pueda desarrollar, el } \\
\text { cual propicia oportunidades } \\
\text { de negocio que puedan } \\
\text { ser explotadas, siempre y } \\
\text { cuando él este a lerta a dichas } \\
\text { oportunidades." (p. 10) } \\
\text { Vínculo empresarial. "Se refiere } \\
\text { a la capacidad de los agentes } \\
\text { para obtener beneficios de sus } \\
\text { estructuras sociales o redes de } \\
\text { pertenencia" (p.10) }\end{array}$ & 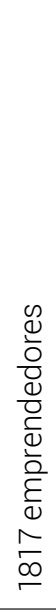 & $\begin{array}{l}\text { Adult } \\
\text { Population } \\
\text { Survey }\end{array}$ & $\begin{array}{l}\text { La percepción de oportunidad fue } \\
\text { determinada por la identidad de género } \\
\text { masculina mientras }(\beta=0,688 ; p=0,05) \text {, } \\
\text { mientras que fue influida por laidentidad } \\
\text { de género femenina }(\beta=0,495 ; p=0,05) \text {. } \\
\text { Respecto al capital social emprendedor, } \\
\text { la identidad masculina incidió en mayor } \\
\text { medida que la identidad de género } \\
\text { femenina }(0,661 ; p=0,05) \text {. Y el miedo al } \\
\text { fraso influyó más en la identidad femenina } \\
(0,448 ; p=, 05) \text {. }\end{array}$ \\
\hline
\end{tabular}

Fuente: Elaboración propia

A partir de las categorías de entrevista y análisis se construyó un cuestionario de preguntas abiertas las cuales permitieron recabar los discursos, significados y sentidos de docentes insertos en la red de conocimiento.

El cuestionario incluye preguntas sociodemográficas (¿cuál es tu edad?, ¿cuál es tu ingreso mensual? En caso de contar con beca ¿cuál es la cantidad que te asignan?, aproximadamente; ¿cuánto es el porcentaje que asignas a tu formación profesional?) y organizacionales (¿perteneces a una red relacionada con tu profesión?, ¿asistes a eventos relativos a tu profesión?, ¿cuáles son los temas y/o problemáticas más comunes en el grupo al que perteneces?, ¿cuál es la tecnología de información más utiliza por el grupo al que perteneces?, ¿cuáles son las funciones relacionadas con esta tecnología?, ¿cuánto es el financiamiento que recibes por el uso intensivo de esta tecnología?) relativas a las cuatro categorías. Las preguntas sociodemográficas incluyen sexo, edad, escolaridad, función e ingreso. Respecto a las preguntas organizacionales, se dividieron en cuatro categorías con el propósito de explorar: cognición, logística, socialización y complejidad advertidas en la revisión de la literatura. 
Procedimiento. Una vez que se construyó la guía se procedió a entrevistar a tres docentes cuya función dentro de la red correspondió a puntos de ventas diferentes. Los tres docentes fueron varones encuestados quienes declaran tener 30, 32 y 36 años cumplidos, poseer el grado de maestría en psicología, administración y sociología, trabajar por asignaturas y horas que corresponden con un salario de 5500 pesos mensuales.

Las entrevistas se llevaron a cabo durante los días 15, 16 y 17 de noviembre de 2011, en promedio las entrevistas duraron de 30 a 50 minutos y se llevaron a cabo en la sucursal correspondiente. Antes de iniciar la entrevista se les preguntó si podían ser grabadas sus declaraciones y en los casos en que se aceptó la condición, se procedió a informar sobre los usos con fines académicos de las entrevistas las cuales no tendrían ninguna consecuencia positiva o negativa en su formación académica o eventual contratación profesional. Al finalizar la entrevista, se les ofreció la posibilidad de consultar los resultados de la investigación vía correo electrónico. Transcurridos unos días les fueron entregados sus reconocimientos por su participación en el estudio.

Análisis. La técnica de síntomas fue empleada para el análisis del discurso, ésta consiste en ordenar extractos de las respuestas a las preguntas expuestas siguiendo una jerarquía de significaciones relativas a las categorías establecidas. Una vez sintetizadas y jerarquizadas en oraciones, las declaraciones son relacionadas considerando sus frecuencias de relación (entrada y salida de información).

Para vaciar y sintetizar la información, se emplearon matrices. Una vez procesada la información, se elaboró la red para facilitar la presentación de los resultados. El software Genopro, versión para estudiantes, permitió la elaboración de redes y sus correspondientes discursos en torno a las categorías de análisis.

\section{RESULTADOS}

La figura 5 muestra las frecuencias relativas a las palabras que vincularon a los agentes (profesores, administradores, estudiantes y practicantes) con la UAEM y NISSAN en tres procesos de gestión, innovación y emprendimiento (véase figura 5). 


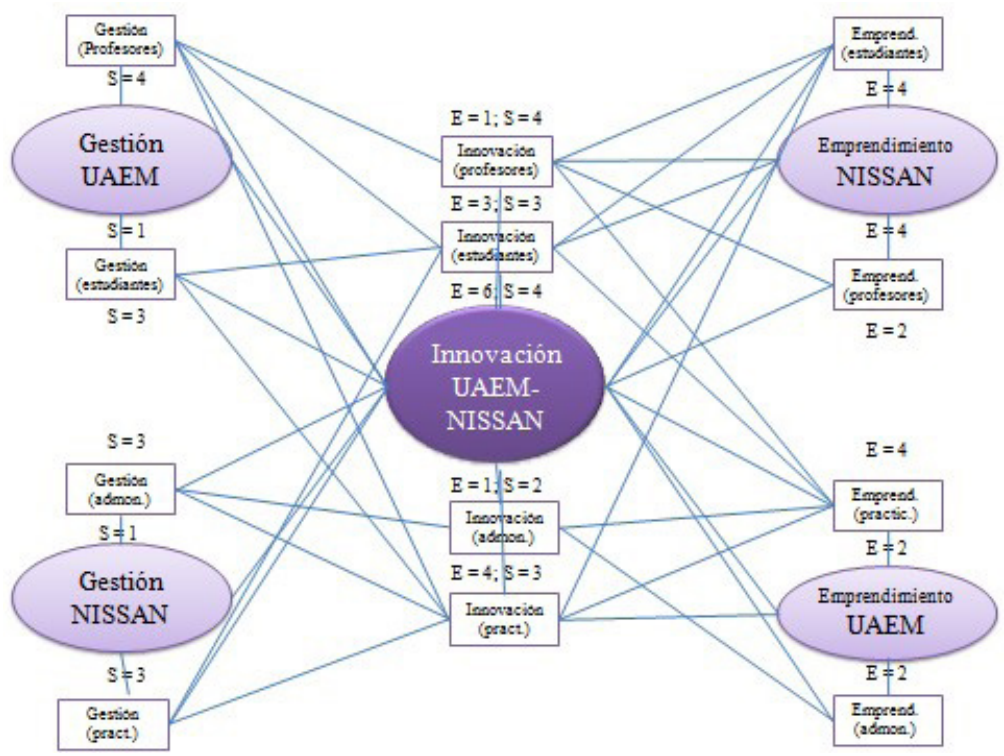

Figura 5. Redes de formación profesional (discursos de profesores) Fuente: Elaborada con los datos del estudio

Los resultados muestran que los discursos enlazan el proceso de gestión con el de innovación (15 entradas de información) que contrastan con sus salidas (18 salidas de información). Es decir, la gestión de profesores y administrativos repercutió en el emprendimiento de estudiantes y practicantes, aunque éstos no influyeron en los otros dos agentes del conocimiento. Ello fue interpretado como una red formativa emergente que depende de la gestión de profesores y administrativos más que de las iniciativas de estudiantes y practicantes de los que se espera una mayor participación.

Cabe destacar que el primer y segundo nodo trans-periférico de entrada de información no establecieron contacto alguno con alguna de las cuatro centrales periféricas (innovación de profesores, administrativos, estudiantes y practicantes), ya que sólo se vincularon con el nodo central (innovación UAEM-NISSAN). 
Precisamente, el balance de entradas y salidas informativas de las centrales periféricas advierte un desbalance en las centrales innovadoras periféricas de estudiantes y practicantes que reciben más información de la que producen ( $E=6$ y $S=4 ; E=4 ; S=3$ respectivamente). En cambio, los profesores cuadruplican la información recibida y los administrativos la duplican $(E=1 ; S=4 ; E$ $=1 ; S=2$ consecutivamente). Es por ello que el balance del nodo central es desfavorable porque recibe más información de la que emite $(E=6 ; S=4)$.

Por consiguiente, la innovación es espuria en la red formativa profesional UAEM-NISSAN con respecto a los esfuerzos de gestión y la emergencia del emprendimiento.

\section{DISCUSIÓN}

El presente estudio ha explorado la emergencia de una red formativa profesional en la que una institución se vincula con una empresa para insertar al mercado laboral a sus estudiantes y futuros egresados. Al formarse la red mediante un convenio de prácticas profesionales, los profesores con experiencia en el campo laboral resultaron los únicos beneficiados debido a que los practicantes se insertan al mercado laboral de un modo previsible, aunque efímero ya que los estudiantes de nueva generación deben pasar por el mismo proceso.

La competencia por un puesto es nula y por ello la innovación está ausente en el sistema de prácticas ya que la reproducción del conocimiento es mayor a su producción o emprendimiento.

Sin embargo, un estudio relativo a las percepciones de autoeficiencia, motivación y compromiso permitirán corroborar los hallazgos expuestos. A medida que la red formativa se consolide, aumentará la competencia por los puestos, la creatividad se incrementará y la innovación emergerá como reguladora del emprendimiento de proyectos.

\section{CONCLUSIÓN}

El aporte del presente estudio al estado del conocimiento radica en que se ha explorado la estructura de una red formativa profesional desde los discursos de profesores involucrados en la capacitación y supervisión de prácticas profesionales. 
A partir de los resultados esgrimidos es menester considerar los discursos de estudiantes, administrativos y practicantes como agentes de conocimiento, pero dado que la red es incipiente, se espera observar el desarrollo de estos agentes una vez que el balance entre la entrada de información y la salida se favorable a la innovación y repercuta en el emprendimiento de proyectos.

\section{REFERENCIAS}

Adenike, A. (2011). Organization climate as a predictor of employee job satisfaction. Business Intelligence Journal. 4, 151-166

Anwar, F. y Norulkamar, U. (2012). Mediating role of organizational commitment among leaderschip and employee outcomes, and empirical evidence from telecom sector. Processing International Seminar on Industrial Engineering and Management 2, 116-161

Arnau, L. y Montané, J. (2010). Aportaciones sobre la relación conceptual entre actitud y competencia, desde la teoría del cambio de actitudes. Journal of Research in Educational Psychology. 8, 1283-1302

Borjas, L. (2010). El espíritu empresarial desde las representaciones sociales: caso Venezuela. Ciencias Sociales, $5,149-165$

Carreón, J. (2013). El desarrollo local sustentable en la esfera ciudadana y comunitaria. Sociedad y Economía, 18 (44), 35-48

Carreón, J. (2014). Prueba empírica de un modelo de establecimiento de agenda. Acta Universitaria, 24 (3), 50-62

Castel, G. y Freundlich, F. (2010). Percepciones de los socios y no socios cooperativista sobre la satisfacción laboral. Revesco. 103, 33-58

Castro, M. y Martins, M. (2010). The relationships between organizational climate and employee satisfaction in information and technology organization. Tydskriff vir Bredyfsielkunde. 36, 1-9 
Cerrón, L. (2010). El papel del mercado en la construcción de los modelos de formación profesional: la mercantilización del sistema. Reifop, 13, 54-63

Chinchilla, N. y Cruz, H. (2010). Diversidad y paradigmas de empresa: un nuevo enfoque. Revista Empresa y Humanismo, 14, 47-79

Coronel, A. (2010). Capacitación del capital humano para una inversión de desarrollo. Eureka, 7, 71-76

Cuesta, A. (2012). Modelo integrado de gestión humana y del conocimiento: una tecnología de aplicación. Revista Venezolana de Gerencia, 57, 86-98

Díaz, S. (2013). Lo humano en la Teoría de las Organizaciones. Visión gerencial, 12, 45-57

Fuentes, F. y Sánchez, S. (2010). Análisis del perfil emprendedor: una perspectiva de género. Estudios de Economía Aplicada, 28, 1-28

Galindo, R. y Echavarría, M. (2011). Diagnóstico de la cultura emprendedora en la escuela de ingeniería de Antioquia. Revista de la Escuela de Ingeniería de Antioquia, 15, 85-94

García, C. (2013). Actitud hacia la utilidad y riesgo en las redes sociales. Folios, 29, 81-93

García, C. (2014). Contraste de un modelo de compromiso laboral en centros de salud pública. Acta Universitaria, 24 (1), 48-59

Gargallo, A. (2010). Percepciones de los socios y no socios cooperativistas sobre la satisfacción laboral. Revesco, 103, 33-58

Gil, C. (2010). Comunicadores corporativos: desafíos de una formación profesional por competencias en la era global. Cuadernos, 33, 49-59

Holden, R. y Karsh, B. (2010). Technology Acceptance Model: its past and its future in health care. Jorunal of Biomedical Informatics. 43, 169-172 
Long, H. (2013). The relationships among learning orientation, market orientation, entrepreneurial orientation, and firm performance. Management Review, 20, 37-46

López, L. y López, J. (2011). Los modelos de adopción de tecnologías de información desde el paradigma actitudinal. Cadernos Ebape. 9, 176-196

Manning, A. (2010). Development of the psychological climate scale for small business. Journal of New Business Ideas \& Trends. 8, 50-63

Medina, C. (2010). Los estudios organizacionales: entre la unidad y la fragmentación. Cinta de Moebio, $38,91-109$

Omar, A. (2010). Liderazgo transformador y satisfacción laboral: el rol de la confianza en el supervisor. Liberabit. 17, 129-137

Orantes, S. (2011). Viabilidad del Modelo de la Aceptación de la Tecnología en las empresas mexicanas. Una aproximación a las actitudes y percepciones de los usuarios de las tecnologías de la información. Revista Digital Universitaria. 12, 1-15

Prada, R. (2013). La adaptación al cambio y el servicio: claves del liderazgo en el mejoramiento de la productividad en las organizaciones. Revista de Estudios Avanzados de Liderazgo, 1, 45-50

Sobrados, L. y Fernández, E. (2010). Competencias emprendedoras y desarrollo del espíritu empresarial en los centros educativos. Educación XXI, 13, 15-38

Tayo, E. y Adeyemi, A. (2012). Job involvement \& organizational commitment as determinants of job performance among educational resource centre personal. European Journal of Globalization and Development Research. 5, 301-313

Vargas, J. (2011). Organización del trabajo y satisfacción laboral: un estudio de caso en la industria del calzado. Revista Electrónica Nova Scientia. 4, 172-204 
Vargas, M. y Arenas, M. (2012). Competencias emprendedoras en estudiantes de psicopedagogía de la Universidad Pedagógica y Tecnológica de Colombia. Revista de Estudios Avanzados de Liderazgo, $1,25-30$

Yuangion, Y. (2011). The impact of strong ties on entrepreneurial intention. An empirical study based on the mediating role of self-efficacy. Journal Entrepreneurship, 3, 147-158

Zampetakis, L. y Moustakis, V. (2013). Entrepreneurial behavior in the Grekk public sector. Emerald, 13, $1-7$ 